\title{
GRS 1758-258 as a winged microquasar
}

\section{Josep Martí*i}

Departamento de Física (EPSJ), Universidad de Jaén, Campus Las Lagunillas s/n Ed. A3, E-23071 Jaén, Spain

E-mail: jmarti@ujaen.es

\section{Pedro L. Luque-Escamilla}

Departamento de Ingeniería Mecánica y Minera (EPSJ), Universidad de Jaén, Campus Las Lagunillas s/n Ed. A3, E-23071 Jaén, Spain

E-mail: peter@ujaen.es

\section{Josep M. Paredes, Valentí Bosch-Ramon}

Departament de Física Quàntica i Astrofísica, Institut de Ciències del Cosmos, Universitat de Barcelona, IEEC-UB, Martí i Franquès 1, E-08028 Barcelona, Spain

E-mail: jmparedeseub.edu, vboschleqa.ub.edu

A deep radio image of the microquasar GRS 1758-258 has evidenced for the first time a new link with the so called 'winged' radio galaxies. This opens a new perspective for the understanding of these secondary lobe features in relativistic jets. The fact that we also find them in a relativistic stellar outflow is better understood in the context of hydrodynamic models. This is in contrast to other alternative interpretations that are mostly based on the spin-flip of the central black hole in radio galaxies.

High Energy Phenomena in Relativistic Outflows VII - HEPRO VII

9-12 July 2019

Facultat de Física, Universitat de Barcelona, Spain

* Speaker.

${ }^{\dagger}$ A footnote may follow. 


\section{Introduction}

GRS 1758-258 is a microquasar originally discovered by the SIGMA coded-mask telescope on board the GRANAT space observatory in the early 90s. [1]. Together with its 'twin' 1E 1740.72942 [2], both sources dominate the Galactic Centre region in hard X-rays. The microquasar concept is widely accepted today as a family of stellar binary systems powered by mass transfer from a normal donor star towards its black hole, or neutron star, companion. Non-thermal plasma jets emerging from the regions close to the compact member of the stellar pair are the most spectacular signature of this mass accretion process, that strongly resembles the accretion-ejection phenomena also seen in active galactic nuclei (AGN) [3,4]. In some cases, these collimated outflows even display superluminal motion effects just as their extragalactic relatives often do [5].

The detection of radio jets in GRS 1758-258 took place soon after the SIGMA discovery and it was possible thanks to interferometric radio observations with the historic Very Large Array (VLA) [6]. This is a facility of the National Radio Astronomy Observatory (NRAO) in New Mexico (USA), currently known as the Karl G. Jansky VLA after undergoing a significant sensitivity upgrade. The VLA also revealed noticeable structural changes in the GRS 1758-258 extended radio jets taking place over time scales of few years [7]. This fact was key to confirm its Milky Way membership and set a distance upper limit of about $12 \mathrm{kpc}$ based on causality arguments [7]. Progress at other wavelengths proceeded at a slower pace over the years, and only after strong observational effort a highly absorbed and variable infrared counterpart could be identified [8,9]. Further spectroscopic analysis with a 10-m class telescope provided hints that GRS 1758-248 is likely an intermediate-mass system with a mid-A spectral type [10]. Unfortunately, even with such a large instrumentation we are still missing good optical and infrared spectra of this source not only because of its weakness but also due to the severely crowded fields in the Galactic Centre vicinity.

In this contribution, we present the results of a deep radio imaging of GRS 1758-258 that resulted after stacking different VLA observing runs acquired over the years. This enabled us to reveal faint extended radio features that enhanced even more the analogy between microquasars and other AGN systems, in particular the so called 'winged' radio galaxies (WRGs) [11, 12]. These are FRII radio galaxies whose jets and lobes display a peculiar X or Z-shaped morphology at large distances from their central core. The origin of these secondary lobe structures is currently a matter of debate since they cannot always be due to simple jet precession effects or double AGN systems. Other very different physical scenarios coexist at present with different degrees of support within the astrophysical community. In particular, the most relevant competing models essentially invoque some kind of spin-flip due to the previous merger of super-massive black holes [13, 14, 15] or, alternatively, a back-flow effect based on hydrodynamic causes [16, 17]. Finding out which is the dominant scenario is key to establish wether or not WRG are actually tracers of supermassive black hole mergers, as this has consequences on the estimated background of gravitational waves in the Universe [12].

In the following sections, we will present a short account of how, unexpectedly, GRS 1758258 turned out to become a sort of missing link between microquasars and the realm of WRGs that may help in the debate outlined above. The reader is referred to [18] for a in depth presentation of the applied methodology and results. 
Table 1: $\log$ of radio observations at $\lambda=6 \mathrm{~cm}$

\begin{tabular}{cccccc}
\hline Project code & VLA Conf. & Date & Duration $(\mathrm{h})$ & Bandwidth (MHz) & Notes \\
\hline 16A-005 & C & 4-22 Mar 2016 & 2.1 & 2048 & Jansky VLA \\
AM560 & C & 3-24 Aug 1997 & 5.8 & 50 & Historic VLA \\
AM428 & CD & 3-4 Oct 1993 & 1.8 & 50 & Historic VLA \\
AM345 & D & 26-27 Sep 1992 & 1.6 & 50 & Historic VLA \\
AM385 & d & 10-11 Sep 1992 & 2.4 & 50 & Historic VLA \\
\hline
\end{tabular}

\section{Observations with the historic and the upgraded VLA}

Table 1 contains the log of Jansky VLA and historic VLA observations used for imaging of GRS $1758-258$ in this work. The instrumental setup of these runs was very different, specially concerning the bandwidth of the oldest and newest ones. This rendered their combined calibration not an easy task. We performed it using the AIPS software package of NRAO as described in [18]. The sources $3 \mathrm{C} 286$ and 3C48 were used both as flux density and bandpass calibrators. Phase calibration relied on the GRS 1758-258 nearby source J1751-253. The final result is presented in Fig. 1 map that illustrates the outcome of merging all Table 1 datasets into a single image. Natural weight and a taper of $25 \mathrm{k} \lambda$ was assigned to all the interferometric visibilities in order to better enhance extended radio emission. It is remarkable that a root-mean-squared (rms) noise as low as $4 \mu \mathrm{Jy}_{\text {beeam }}{ }^{-1}$ was achieved that improves over the sensitivity of previously published maps [7].

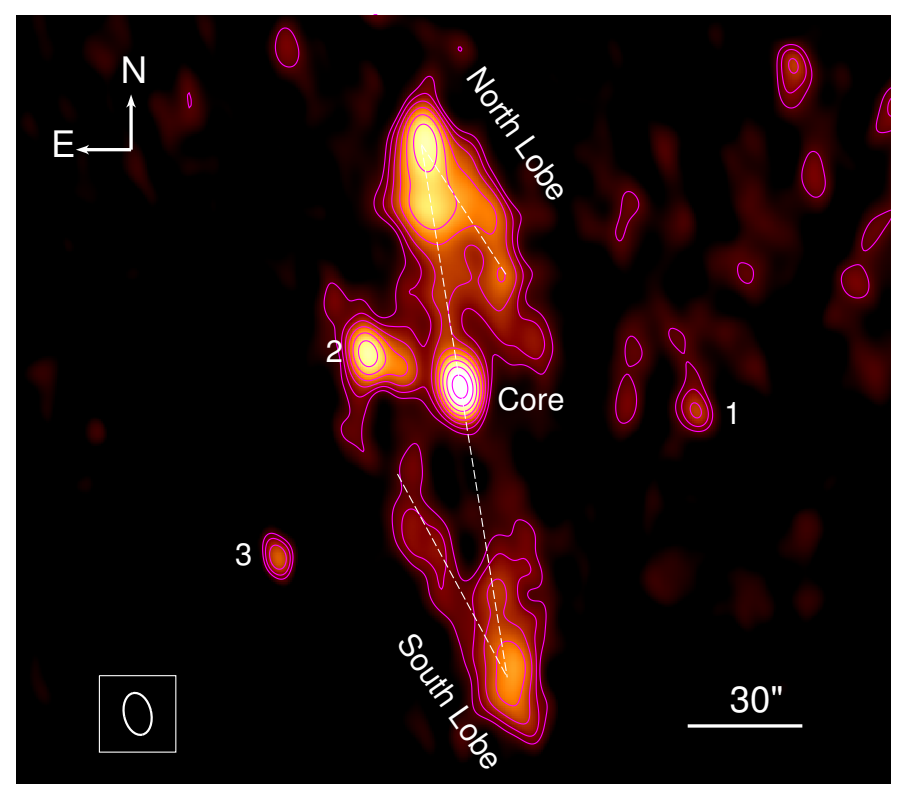

Figure 1: Deep radio map of GRS 1758-258 obtained by combining all Table 1 data analyzed in this work. Contours start at four times the rms noise of $4 \mu \mathrm{Jy} \mathrm{beam}^{-1}$ and proceed in $\sqrt{2}$ steps. The lower-left ellipse displays the full-width half maximum of the synthesized beam that corresponds to $11.3 \times 7.2$ arc-second $^{2}$ with position angle of $13^{\circ}$. The angular scale is given by horizontal bar at the lower-right corner. The main radio features are labeled and a few background radio source numbered from 1 to 3 . The apparent Z-like morphology of extended emission features is outlined by a dashed line. 


\section{Discussion}

The most remarkable fact in Fig. 1 is the presence of different extended emission features. First, the main bipolar jet flow emanating from the GRS 1758-258 central core and terminating at the microquasar northern and southern lobes, respectively. Second, the two bridges of extended emission that originate at each these lobes and, apparently, stream back towards the core vicinity. The overall path of their ensemble strongly resembles a Z-like morphology that is strongly evocative of WRGs. This is the first time that such extended radio wings are detected in a microquasar. Therefore, the radio image in Fig. 1 reinforces even more the widely accepted similarity of microquasars with extragalactic sources of relativistic jets. We anticipate here that comparable wing features are naturally expected to exist in other microquasars, in particular in the GRS 1758-258 'twin' 1E 1740.7-2942. However, we foresee them to be very low surface brightness sources and clear evidence for them will likely need to wait for the future generation of radio interferometers such as the Square Kilometer Array (SKA).

Having found wings in GRS 1758-248, the obvious next question is how did they form? Black hole merger scenarios with spin-flip clearly do not apply in this case. The reason is simply because conventional stellar evolution dictates that only one compact object is expected to exist in a microquasar binary system. Also, the estimated re-alignment time scale $(\sim 1 \mathrm{Myr})$ is unrealistically larger than the estimated jet travel time and age $\left(10^{3}-10^{5} \mathrm{yr}\right)$ for a $10 M_{\odot}$ black hole [18]. The alternative scenario that better fits the GRS 1758-258 case is one of hydrodynamic back-flow-type [18], with a light jet colliding with its ambient medium. This goes along the lines proposed in some previous WRG works such as [19] and [20]. A key point to support this interpretation comes from the Dame's CO survey of the Galactic Plane [21], where a conspicuous CO gas cloud is found towards GRS 1758-258. Moreover, the cloud's Local Standard of Rest (LSR) velocity is $v_{\text {LSR }} \simeq 210$ $\mathrm{km} \mathrm{s}^{-1}$, a value just kinematically consistent with the $8.5 \mathrm{kpc}$ distance usually assumed for GRS 1758-258 based on its Galactic Center vicinity. Differences in brightness between the northern and southern lobe and wing structures are naturally attributed to a likely asymmetric density distribution within the gas cloud.

\section{Conclusions}

A deep radio imaging of GRS 1758-258 teaches us that this microquasar behaves as their extragalactic relatives in a new and unexpected way: the formation of extended wings. Finding these structures in an environment different than WRGs allows us to study them within the framework of stellar evolution, that excludes some of the physical scenarios proposed for extragalactic sources, in particular those based on black hole spin-flip. Thanks to this new perspective, we conclude that wings can be formed in a stellar microquasar by a purely hydrodynamic back-flow mechanism. Extrapolating this finding to the extragalactic universe, we infer that some WRGs could also form in this way. Of course, alternative formation scenarios cannot strictly ruled out in all cases but, definitely, we can no longer consider WRGs as entirely reliable tracers of black hole merger events. Finally, estimates of the gravitational wave background associated with WRGs should take into account this caution warning. 


\section{Acknowledgements}

The National Radio Astronomy Observatory is a facility of the National Science Foundation operated under cooperative agreement by Associated Universities, Inc. This work was supported by the Agencia Estatal de Investigación grants AYA2016-76012-C3-1-P and AYA2016-76012-C33-P from the Spanish Ministerio de Economía y Competitividad (MINECO), by Consejería de Economía, Innovación, Ciencia y Empleo of Junta de Andalucía under research group FQM-322, by grant MDM-2014-0369 of the ICCUB (Unidad de Excelencia 'María de Maeztu'), and by the Catalan DEC grant 2017 SGR 643, as well as FEDER funds.

\section{References}

[1] R. Sunyaev, M. Gilfanov, E. Churazov, M. Pavlinsky, G. Babalyan, I. Dekhanov et al., Two hard $X$-ray sources in the field of the galactic center : well known 1E 1740.7-2942 and discovered GRS 1758-258., Pisma v Astronomicheskii Zhurnal 17 (1991) 116.

[2] I. F. Mirabel, L. F. Rodríguez, B. Cordier, J. Paul and F. Lebrun, A double-sided radio jet from the compact Galactic Centre annihilator 1E1740.7-2942, Nature 358 (1992) 215.

[3] I. F. Mirabel and L. F. Rodríguez, Sources of Relativistic Jets in the Galaxy, Annual Review of Astron and Astrophys 37 (1999) 409 [astro-ph/9902062].

[4] S. Corbel, Microquasars: an observational review., in Jets at All Scales, G. E. Romero, R. A. Sunyaev and T. Belloni, eds., vol. 275 of IAU Symposium, pp. 205-214, Feb, 2011, DOI.

[5] I. F. Mirabel and L. F. Rodríguez, A superluminal source in the Galaxy, Nature 371 (1994) 46.

[6] L. F. Rodríguez, I. F. Mirabel and J. Martí, The Radio Counterpart of the Hard X-Ray Source GRS 1758-258, Astrophysical Journal, Letters 401 (1992) L15.

[7] J. Martí, P. L. Luque-Escamilla, G. E. Romero, J. R. Sánchez-Sutil and Á. J. Muñoz-Arjonilla, Real-time evolution of a large-scale relativistic jet, Astronomy and AstrophysicAstronomy and Astrophysicss 578 (2015) L11 [1505.07641].

[8] A. J. Muñoz-Arjonilla, J. Martí, P. L. Luque-Escamilla, J. R. Sánchez-Sutil, E. Sánchez-Ayaso, J. A. Combi et al., Identification of the optical and near-infrared counterpart of GRS 1758-258, Astronomya and Astrophysics 519 (2010) A15 [1006.4954].

[9] P. L. Luque-Escamilla, J. Martí and Á. J. Muñoz-Arjonilla, The Variable Near-infrared Counterpart of the Microquasar GRS 1758-258, Astrophysical Journal, Letters 797 (2014) L1.

[10] J. Martí, P. L. Luque-Escamilla and Á. J. Muñoz-Arjonilla, Optical spectroscopy of the microquasar GRS 1758-258: a possible intermediate mass system?, Astronomy and Astrophysics 596 (2016) A46 [1611.03683].

[11] C. C. Cheung, FIRST “Winged” and X-Shaped Radio Source Candidates, Astronomical Journal 133 (2007) 2097 [astro-ph/0701278].

[12] L. Saripalli and R. Subrahmanyan, The Genesis of Morphologies in Extended Radio Sources: $X$-Shapes, Off-Axis Distortions, and Giant Radio Sources, Astrophysical Journal, Letters 695 (2009) 156 [0811.1907].

[13] D. Merritt and R. D. Ekers, Tracing Black Hole Mergers Through Radio Lobe Morphology, Science 297 (2002) 1310 [astro-ph/ 0208001 ]. 
[14] J. Dennett-Thorpe, P. A. G. Scheuer, R. A. Laing, A. H. Bridle, G. G. Pooley and W. Reich, Jet reorientation in active galactic nuclei: two winged radio galaxies, Monthly Notices of the RAS 330 (2002) 609 [astro-ph/0110339].

[15] L. Á. Gergely and P. L. Biermann, The Spin-Flip Phenomenon in Supermassive Black hole binary mergers, Astrophysical Journal, Letters 697 (2009) 1621 [0704.1968].

[16] A. Capetti, S. Zamfir, P. Rossi, G. Bodo, C. Zanni and S. Massaglia, On the origin of X-shaped radio-sources: New insights from the properties of their host galaxies, Astronomy and Astrophysics 394 (2002) 39 [astro-ph/0207333].

[17] E. J. Hodges-Kluck and C. S. Reynolds, Hydrodynamic Models of Radio Galaxy Morphology: Winged and X-shaped Sources, Astrophysical Journal, Letters 733 (2011) 58 [1103 . 4863].

[18] J. Martí, P. L. Luque-Escamilla, V. Bosch-Ramon and J. M. Paredes, A galactic microquasar mimicking winged radio galaxies, Nature Communications 8 (2017) 1757.

[19] Gopal-Krishna, P. L. Biermann and P. J. Wiita, The Origin of X-shaped Radio Galaxies: Clues from the Z-symmetric Secondary Lobes, Astrophysical Journal, Letters 594 (2003) L103 [astro-ph/0308059].

[20] Gopal-Krishna, P. L. Biermann, L. Á. Gergely and P. J. Wiita, On the origin of X-shaped radio galaxies, Research in Astronomy and Astrophysics 12 (2012) 127 [1008. 0789].

[21] T. M. Dame, D. Hartmann and P. Thaddeus, The Milky Way in Molecular Clouds: A New Complete CO Survey, Astrophysical Journal, Letters 547 (2001) 792 [astro-ph/ 0009217 ]. 